\title{
THE AHARONOV-BOHM HAMILTONIAN WITH TWO VORTICES REVISITED
}

\author{
Petra KošŤÁKová, PAVEL ŠŤOvíČEK* \\ Department of Mathematics, Faculty of Nuclear Sciences and Physical Engineering, Czech Technical University \\ in Prague, Trojanova 13, 12000 Praha, Czech Republic \\ * corresponding author: stovipav@kmlinux.fjfi.cvut.cz
}

\begin{abstract}
We consider an invariant quantum Hamiltonian $H=-\Delta_{L B}+V$ in the $L^{2}$ space based on a Riemannian manifold $\tilde{M}$ with a discrete symmetry group $\Gamma$. To any unitary representation $\Lambda$ of $\Gamma$ one can relate another operator on $M=\tilde{M} / \Gamma$, called $H_{\Lambda}$, which formally corresponds to the same differential operator as $H$ but which is determined by quasi-periodic boundary conditions. As originally observed by Schulman in theoretical physics and Sunada in mathematics, one can construct the propagator associated with $H_{\Lambda}$ provided one knows the propagator associated with $H$. This approach is reviewed and demonstrated on a quantum model describing a charged particle on the plane with two Aharonov-Bohm vortices. The construction of the propagator is explained in full detail including all substantial intermediate steps.
\end{abstract}

KEYworDs: Aharonov-Bohm effect; propagator; covering space; Bloch decomposition.

\section{IntRoduCtion}

Suppose there is given a Riemannian manifold $\tilde{M}$ with a discrete symmetry group $\Gamma$ and a $\Gamma$-periodic Hamilton operator $H$ on $L^{2}(\tilde{M})$. To any unitary representation $\Lambda$ of $\Gamma$ one can relate another operator on $M=\tilde{M} / \Gamma$, called $H_{\Lambda}$, which is determined by quasi-periodic boundary conditions. A formula relating the propagators $\mathcal{K}_{t}^{\Lambda}\left(x, x_{0}\right)$ and $\mathcal{K}_{t}\left(x, x_{0}\right)$ associated with $H_{\Lambda}$ and $H$, respectively, has been derived in the framework of the Feynman path integral [18, 19]. An analogous formula is also known for heat kernels [4]. An opposite point of view is taken when one decomposes the operator $H$ into a direct integral with components $H_{\Lambda}$ where $\Lambda$ runs over all irreducible unitary representations of $\Gamma[3,6,23$. The evolution operator then decomposes correspondingly. This type of decomposition is a substantial step in the Bloch analysis.

The both relations, the propagator formula on one hand and the generalized Bloch decomposition on the other hand, are in a sense mutually inverse [11, 12]. In the current paper we wish to demonstrate how this relationship can be effectively used on a concrete example of interest. We consider the formula for propagators in the case of the Aharonov-Bohm effect with two vortices. In this quantum model, $\tilde{M}$ is identified with the universal covering space of the plane with two excluded points and $\Gamma$ is the fundamental group of the same manifold.

This problem has already been treated by one of the authors quite a long time ago in [21. But the topic is in no way exhausted completely, and this quantum model was intensively discussed in a number of papers, in some cases even very recently. These discussions rely on completely different approaches, however, like asymptotic methods for largely separated vortices, semiclassical analysis and a complex scaling method [1, 2, 9, 25]. One may also mention more complex models comprising, apart of magnetic vortices, also additional potentials or magnetic fields [14, 16, or models with an arbitrary finite number of magnetic vortices or even with countably many vortices arranged in a lattice [15, 17, 22]. On the other hand, the method stemming from the original ideas of Schulman and Sunada turned out to be fruitful also in analysis of other interesting models like Brownian random walk on the twice punctured plane [5, 7].

Here we return to the article [21] which is in its character a brief letter presenting the final formulas without a detailed derivation. But the technique applied therein is of independent interest and can prove useful in other situations as well, as already mentioned above. This is why we focus, in the present paper, primarily on the method itself and aim to explain the approach on a concrete example while indicating all necessary intermediate steps in full detail. Hopefully, the provided analysis may open the way to new applications of the method.

The paper is organized as follows. The main ideas and results of the general approach are outlined in Section 2 following papers [11, 12. Section 3 is the key section of the present paper. In Subsection 3.1, a formula for the propagator on the universal covering space of the twice punctured plane, as originally presented in [21, is briefly recalled. Subsection 3.2 has a preliminary character and provides a summary of some auxiliary useful identities. Subsection 3.3 is fully dedicated to the proof of the propagator formula, as given in (9), (10). More precisely, the goal of this subsection is a verification of equation (17). As a corollary, in Section 4 more details 
are provided, if compared to [21], about a formal application of the Schulman-Sunada formula, as recalled in (5), to the studied example while making use of the knowledge of the propagator on the covering space.

\section{A SUMmaRY OF THE GENERAL APPROACH}

\subsection{Periodic Hamiltonians}

Let $\tilde{M}$ be a connected Riemannian manifold with a discrete and at most countable symmetry group $\Gamma$. The action of $\Gamma$ on $\tilde{M}$ is assumed to be smooth, free and proper. Let us recall that under these assumptions any element $s \in \Gamma$ different from the unity has no fixed points on $\tilde{M}$, and for any compact set $K \subset \tilde{M}$, the intersection $K \cap s \cdot K$ is nonempty for at most finitely many elements $s \in \Gamma$. This also implies that any point $y \in \tilde{M}$ has a neighborhood $U$ such that the sets $s \cdot U, s \in \Gamma$, are mutually disjoint [13, Corollary 12.10].

Denote by $\tilde{\mu}$ the measure on $\tilde{M}$ induced by the Riemannian metric. The quotient $M=\tilde{M} / \Gamma$ is a connected Riemannian manifold with an induced measure $\mu$. This way one gets a principal fiber bundle $\pi: \tilde{M} \rightarrow M$ with the structure group $\Gamma$. All $L^{2}$ spaces based on the manifolds $M$ and $\tilde{M}$ are everywhere tacitly understood with the measures $\mu$ and $\tilde{\mu}$, respectively.

In a number of important examples, $\tilde{M}$ is the universal covering space of $M$ and $\Gamma=\pi_{1}(M)$ is the fundamental group of $M$. In particular, this is the case when one is considering the Aharonov-Bohm effect.

To a unitary representation $\Lambda$ of $\Gamma$ in a separable Hilbert space $\mathscr{L}_{\Lambda}$ one relates the Hilbert space $\mathscr{H}_{\Lambda}$ formed by $\Lambda$-equivariant vector-valued functions on $\tilde{M}$. This means that any function $\psi \in \mathscr{H}_{\Lambda}$ is measurable with values in $\mathscr{L}_{\Lambda}$ and satisfies

$$
\forall s \in \Gamma, \psi(s \cdot y)=\Lambda(s) \psi(y) \text { almost everywhere on } \tilde{M} \text {. }
$$

Moreover, the norm of $\psi$ induced by the following scalar product is required to be finite. If $\psi_{1}, \psi_{2} \in \mathscr{H}_{\Lambda}$ then the function $y \mapsto\left\langle\psi_{1}(y), \psi_{2}(y)\right\rangle_{\mathscr{L}_{\Lambda}}$ defined on $\tilde{M}$ is $\Gamma$-invariant and so it projects to a function $s_{\psi_{1}, \psi_{2}}$ defined on $M$, and the scalar product is defined by

$$
\left\langle\psi_{1}, \psi_{2}\right\rangle=\int_{M} s_{\psi_{1}, \psi_{2}}(x) \mathrm{d} \mu(x) .
$$

Our discussion focuses on $\Gamma$-periodic Hamiltonians on $\tilde{M}$ of the form $H=-\Delta_{L B}+V$ where $\Delta_{L B}$ is the Laplace-Beltrami operator and $V$ is a $\Gamma$-invariant semibounded and locally integrable real function on $\tilde{M}$. Clearly, the differential operator $-\Delta_{L B}+V$ is semibounded on the domain formed by test functions (i.e. smooth and compactly supported functions), and $H$ is defined as its Friedrichs extension.

The same choice will also be made in other instances below in the paper. This is to say that in the presented approach we distinguish the Friedrichs extension as the preferred self-adjoint extension of a given semibounded symmetric operator. Here we are referring to the widely used result ensuring the existence of an unambiguously defined and in some sense minimal self-adjoint extension of a semibounded symmetric operator, the so called Friedrichs extension [10, §VI.2]. This choice is encountered very frequently in various applications and it also makes it possible to avoid the discussion of the domain of the self-adjoint operator in question which sometimes may be quite tedious.

To the same differential operator, $-\Delta_{L B}+V$, one can relate a self-adjoint operator $H_{\Lambda}$ in the space $\mathscr{H}_{\Lambda}$ for any unitary representation $\Lambda$ of $\Gamma$ in $\mathscr{L}_{\Lambda}$. Let us define

$$
\Phi_{\Lambda}: C_{0}^{\infty}(\tilde{M}) \otimes \mathscr{L}_{\Lambda} \rightarrow \mathscr{H}_{\Lambda}
$$

by

$$
\forall \varphi \in C_{0}^{\infty}(\tilde{M}), \forall v \in \mathscr{L}_{\Lambda},\left(\Phi_{\Lambda} \varphi \otimes v\right)(y)=\sum_{s \in \Gamma} \varphi(s \cdot y) \Lambda\left(s^{-1}\right) v
$$

Since the action of $\Gamma$ is proper, the vector-valued function $\Phi_{\Lambda} \varphi \otimes v$ is smooth. Moreover, $\Phi_{\Lambda} \varphi \otimes v$ is $\Lambda$-equivariant, the norm of $\Phi_{\Lambda} \varphi \otimes v$ in $\mathscr{H}_{\Lambda}$ is finite, and the range of $\Phi_{\Lambda}$ is dense in $\mathscr{H}_{\Lambda}$. The Laplace-Beltrami operator is well defined on $\operatorname{Ran}\left(\Phi_{\Lambda}\right)$ and it holds

$$
\Delta_{L B} \Phi_{\Lambda}[\varphi \otimes v]=\Phi_{\Lambda}\left[\Delta_{L B} \varphi \otimes v\right]
$$

One can also verify that the differential operator $-\Delta_{L B}$ is positive on the domain $\operatorname{Ran}\left(\Phi_{\Lambda}\right) \subset \mathscr{H}_{\Lambda}$. Since the function $V(y)$ is $\Gamma$-invariant, the multiplication operator by $V$ is well defined in the Hilbert space $\mathscr{H}_{\Lambda}$. The Hamiltonian $H_{\Lambda}$ is defined as the Friedrichs extension of the differential operator $-\Delta_{L B}+V$ considered on the domain $\operatorname{Ran} \Phi_{\Lambda}$. The reader is referred to [11, 12] for more details. 


\subsection{A generalization of the Bloch Decomposition}

Let $\hat{\Gamma}$ be the dual space to $\Gamma$ (the quotient space of the space of irreducible unitary representations of $\Gamma$ ). In the first step of the generalized Bloch analysis one decomposes $H$ into a direct integral over $\hat{\Gamma}$ with components being equal to $H_{\Lambda}$. To achieve this goal a well defined harmonic analysis on $\Gamma$ is necessary.

It is known that the harmonic analysis is well established for locally compact groups of type I [20]. So all formulas presented below are well defined provided $\Gamma$ is a type I group. As shown in [26, Satz 6], a countable discrete group is of type I if and only if it has an Abelian normal subgroup of finite index. This means that there exist multiply connected configuration spaces of interest whose fundamental groups are not of type I. For example, the fundamental group in the case of the Aharonov-Bohm effect with two vortices is the free group with two generators, and it is not of type I. This problem is avoided, however, if $\tilde{M}$ is the maximal Abelian covering of $M$ rather than the universal covering [12, 24,

Let us recall basic properties of the harmonic analysis on discrete type I groups [20]. The Haar measure on $\Gamma$ is simply the counting measure. Let $\mathrm{d} \hat{m}$ be the Plancherel measure on $\hat{\Gamma}$. It is known that if $\Gamma$ is a countable discrete group of type I then $\operatorname{dim} \mathscr{L}_{\Lambda}$, the dimension of the carrier representation space, is a bounded function of $\Lambda \in \hat{\Gamma}$ [26, Korollar I]. Denote by $\mathscr{I}_{2}\left(\mathscr{L}_{\Lambda}\right) \equiv \mathscr{L}_{\Lambda} \otimes \mathscr{L}_{\Lambda}^{*}$ the Hilbert space formed by Hilbert-Schmidt operators on $\mathscr{L}_{\Lambda}\left(\mathscr{L}_{\Lambda}^{*}\right.$ is the dual space to $\left.\mathscr{L}_{\Lambda}\right)$.

The Fourier transform is defined as a unitary mapping

$$
\mathscr{F}: L^{2}(\Gamma) \rightarrow \int_{\hat{\Gamma}}^{\oplus} \mathscr{I}_{2}\left(\mathscr{L}_{\Lambda}\right) \mathrm{d} \hat{m}(\Lambda) .
$$

Note that in this situation $f \in L^{1}(\Gamma)$ just means that the values of $f$ on $\Gamma$ represent a summable sequence. Since every summable sequence is also square summable we have $f \in L^{1}(\Gamma) \subset L^{2}(\Gamma)$, and then

$$
\mathscr{F}[f](\Lambda)=\sum_{s \in \Gamma} f(s) \Lambda(s) .
$$

Conversely, if $f$ is of the form $f=g * h$ (the convolution) where $g, h \in L^{1}(\Gamma)$, and $\hat{f}=\mathscr{F}[f]$ then

$$
f(s)=\int_{\hat{\Gamma}} \operatorname{Tr}\left[\Lambda(s)^{*} \hat{f}(\Lambda)\right] \mathrm{d} \hat{m}(\Lambda) .
$$

Using unitarity of the Fourier transform one finds that $\hat{m}(\hat{\Gamma}) \leq 1$. The following rule is of crucial importance:

$$
\forall s \in \Gamma, \forall f \in L^{2}(\Gamma), \mathscr{F}[f(s \cdot g)](\Lambda)=\Lambda\left(s^{-1}\right) \mathscr{F}[f(g)](\Lambda) .
$$

Now we are going to construct a unitary map

$$
\Phi: L^{2}(\tilde{M}) \rightarrow \int_{\hat{\Gamma}}^{\oplus} \mathscr{H}_{\Lambda} \otimes \mathscr{L}_{\Lambda}^{*} \mathrm{~d} \hat{m}(\Lambda)
$$

making it possible to decompose $H$. Observe that the tensor product $\mathscr{H}_{\Lambda} \otimes \mathscr{L}_{\Lambda}^{*}$ can be naturally identified with the Hilbert space of $\Lambda$-equivariant operator-valued functions on $\tilde{M}$ with values in $\mathscr{I}_{2}\left(\mathscr{L}_{\Lambda}\right)$. For $f \in L^{2}(\tilde{M})$ and $y \in \tilde{M}$ set

$$
\forall s \in \Gamma, f_{y}(s)=f\left(s^{-1} \cdot y\right)
$$

The norm $\left\|f_{y}\right\|$ in $L^{2}(\Gamma)$ is a $\Gamma$-invariant function of $y \in \tilde{M}$ whose projection onto $M$ is square integrable. Hence for almost all $x \in M$ and all $y \in \pi^{-1}(\{x\})$ one has $f_{y} \in L^{2}(\Gamma)$. We define components $\Phi[f](\Lambda), \Lambda \in \hat{\Gamma}$, by

$$
(\Phi[f](\Lambda))(y)=\mathscr{F}\left[f_{y}\right](\Lambda) \in \mathscr{I}_{2}\left(\mathscr{L}_{\Lambda}\right) .
$$

In particular, if $f \in L^{1}(\tilde{M}) \cap L^{2}(\tilde{M})$ then

$$
(\Phi[f](\Lambda))(y)=\sum_{s \in \Gamma} f\left(s^{-1} \cdot y\right) \Lambda(s) .
$$

Equivalently, referring to (1), one can define $\Phi$ in the following way. For $\varphi \in C_{0}^{\infty}(\tilde{M}), v \in \mathscr{L}_{\Lambda}$ and $y \in \tilde{M}$ set

$$
(\Phi[\varphi](\Lambda))(y) v=\left(\Phi_{\Lambda} \varphi \otimes v\right)(y)
$$

Then $\Phi$ introduced in (2) is an isometry and extends unambiguously to a unitary mapping.

Finally one can verify the formula

$$
\Phi H \Phi^{-1}=\int_{\hat{\Gamma}}^{\oplus} H_{\Lambda} \otimes 1 \mathrm{~d} \hat{m}(\Lambda)
$$

which represents the sought Bloch decomposition. As a corollary we have

$$
\Phi U(t) \Phi^{-1}=\int_{\hat{\Gamma}}^{\oplus} U_{\Lambda}(t) \otimes 1 \mathrm{~d} \hat{m}(\Lambda) .
$$




\subsection{Propagators associated with periodic Hamiltonians}

In (3), the evolution operator $U(t)$ is expressed in terms of $U_{\Lambda}(t), \Lambda \in \hat{\Gamma}$. It is possible to invert this relationship and to derive a formula for the propagator associated with $H_{\Lambda}$ which is expressed in terms of the propagator associated with $H$.

The propagators are regarded as distributions which are introduced as kernels of the corresponding evolution operators. Recall that, by the Schwartz kernel theorem (see, for example, [8, Theorem 5.2.1]), to every $B \in \mathscr{B}\left(L^{2}(\tilde{M})\right)$ there exists one and only one $\beta \in \mathscr{D}^{\prime}(\tilde{M} \times \tilde{M})$ such that

$$
\forall \varphi_{1}, \varphi_{2} \in C_{0}^{\infty}(\tilde{M}), \quad \beta\left(\overline{\varphi_{1}} \otimes \varphi_{2}\right)=\left\langle\varphi_{1}, B \varphi_{2}\right\rangle
$$

One calls $\beta$ the kernel of $B$.

The kernel theorem can be extended to Hilbert spaces formed by $\Lambda$-equivariant vector-valued functions. In this case the kernels are operator-valued distributions. To every $B \in \mathscr{B}\left(\mathscr{H}_{\Lambda}\right)$ there exists one and only one $\beta \in \mathscr{D}^{\prime}(\tilde{M} \times \tilde{M}) \otimes \mathscr{B}\left(\mathscr{L}_{\Lambda}\right)$ such that $\forall \varphi_{1}, \varphi_{2} \in C_{0}^{\infty}(\tilde{M}), \forall v_{1}, v_{2} \in \mathscr{L}_{\Lambda}$,

$$
\left\langle v_{1}, \beta\left(\overline{\varphi_{1}} \otimes \varphi_{2}\right) v_{2}\right\rangle_{\mathscr{L}_{\Lambda}}=\left\langle\Phi_{\Lambda} \varphi_{1} \otimes v_{1}, B \Phi_{\Lambda} \varphi_{2} \otimes v_{2}\right\rangle
$$

The distribution $\beta$ is $\Lambda$-equivariant:

$$
\forall s \in \Gamma, \beta\left(s \cdot y_{1}, y_{2}\right)=\Lambda(s) \beta\left(y_{1}, y_{2}\right) \text { and } \beta\left(y_{1}, s \cdot y_{2}\right)=\beta\left(y_{1}, y_{2}\right) \Lambda\left(s^{-1}\right)
$$

Denote by $\mathcal{K}_{t} \in \mathscr{D}^{\prime}(\tilde{M} \times \tilde{M})$ the kernel of $U(t) \in \mathscr{B}\left(L^{2}(\tilde{M})\right)$, and by $\mathcal{K}_{t}^{\Lambda} \in \mathscr{D}^{\prime}(\tilde{M} \times \tilde{M}) \otimes \mathscr{B}\left(\mathscr{L}_{\Lambda}\right)$ the kernel of $U_{\Lambda}(t) \in \mathscr{B}\left(\mathscr{H}_{\Lambda}\right)$. Here and everywhere in this section, $t$ is a real parameter. The kernel $\mathcal{K}_{t}^{\Lambda}$ is $\Lambda$-equivariant in the sense of $(4)$.

First, we can rewrite the Bloch decomposition $(3)$ in terms of kernels. For all $\varphi_{1}, \varphi_{2} \in C_{0}^{\infty}(\tilde{M})$,

$$
\mathcal{K}_{t}\left(\varphi_{1} \otimes \varphi_{2}\right)=\int_{\hat{\Gamma}} \operatorname{Tr}\left[\mathcal{K}_{t}^{\Lambda}\left(\varphi_{1} \otimes \varphi_{2}\right)\right] \mathrm{d} \hat{m}(\Lambda),
$$

with the integral being convergent. An inverse relation was derived by Schulman in the framework of path integration [18, 19] and reads

$$
\mathcal{K}_{t}^{\Lambda}(x, y)=\sum_{s \in \Gamma} \Lambda(s) \mathcal{K}_{t}\left(s^{-1} \cdot x, y\right)
$$

It is possible to give (5) the following rigorous interpretation [11, 12]. Suppose that $\varphi_{1}, \varphi_{2} \in C_{0}^{\infty}(\tilde{M})$ are fixed but otherwise arbitrary. Set

$$
\begin{aligned}
F_{t}(s) & =\mathcal{K}_{t}\left(\varphi_{1}\left(s^{-1} \cdot y_{1}\right) \otimes \varphi_{2}\left(y_{2}\right)\right) \text { for } s \in \Gamma, \\
G_{t}(\Lambda) & =\mathcal{K}_{t}^{\Lambda}\left(\varphi_{1} \otimes \varphi_{2}\right) \in \mathscr{I}_{2}\left(\mathscr{L}_{\Lambda}\right) \text { for } \Lambda \in \hat{\Gamma} .
\end{aligned}
$$

One can show that $F_{t} \in L^{2}(\Gamma)$ and $G_{t}$ is bounded on $\hat{\Gamma}$ in the Hilbert-Schmidt norm. Recalling that $\hat{m}(\hat{\Gamma}) \leq 1$ we have $\left\|G_{t}(\cdot)\right\| \in L^{1}(\hat{\Gamma}) \cap L^{2}(\hat{\Gamma})$. Then $F_{t}=\mathscr{F}^{-1}\left[G_{t}\right]$ and, consequently,

$$
G_{t}=\mathscr{F}\left[F_{t}\right] .
$$

Rewriting (6) formally yields (5).

\section{The PRopagator ON THE UNIVERSAL COVERING SPACE}

\subsection{A FORMUla FOR THE PROPAGATOR}

The configuration space for the Aharonov-Bohm effect with two vortices is the plane with two excluded points, $M=\mathbb{R}^{2} \backslash\{a, b\}$. This is a flat Riemannian manifold and the same is true for the universal covering space $\tilde{M}$. Let $\pi: \tilde{M} \rightarrow M$ be the projection. It is convenient to complete the manifold $\tilde{M}$ by a countable set of points $\mathcal{A} \cup \mathcal{B}$ lying on the border of $\tilde{M}$ and projecting onto the excluded points, $\pi(\mathcal{A})=\{a\}$ and $\pi(\mathcal{B})=\{b\}$.

$\tilde{M}$ looks locally like $\mathbb{R}^{2}$ but differs from the Euclidean space by some global features. First of all, not every two points from $\tilde{M}$ can be connected by a geodesic segment. Fix a point $x \in \tilde{M}$. The symbol $D(x)$, as introduced below in 20 , stands for the set of points $y \in \tilde{M}$ which can be connected with $x$ by a segment. Then $D(x)$ is a sheet of the covering $\tilde{M} \rightarrow M$. It can be identified with $\mathbb{R}^{2}$ cut along two half-lines with the limit points $a$ and $b$, respectively. The border $\partial D(x)$ is formed by four half-lines. The universal covering space $\tilde{M}$ can be imagined as a result of an infinite process of gluing together countably many copies of $D(x)$ with each copy having four neighbors. 
The fundamental group of $M$, called $\Gamma$, is known to be the free group with two generators $g_{a}$ and $g_{b}$. For the generator $g_{a}$ one can choose the homotopy class of a simple positively oriented loop winding once around the point $a$ and leaving the point $b$ in the exterior. Analogously one can choose $g_{b}$ by interchanging the role of $a$ and $b$. One-dimensional unitary representations $\Lambda$ of $\Gamma$ are determined by two numbers $\alpha, \beta, 0 \leq \alpha$, $\beta<1$, so that

$$
\Lambda\left(g_{a}\right)=e^{2 \pi i \alpha}, \Lambda\left(g_{b}\right)=e^{2 \pi i \beta} .
$$

The standard way how to define the Aharonov-Bohm Hamiltonian $H_{A B}$ with two vortices is to choose a vector potential $\vec{A}$ for which curl $\vec{A}=0$ on $M$ and such that the nonintegrable phase factor [27] for a closed path from the homotopy class $g_{a}$ or $g_{b}$ equals $e^{2 \pi i \alpha}$ or $e^{2 \pi i \beta}$, respectively (assuming that $0<\alpha, \beta<1$ ). $H_{A B}$ then acts as the differential operator $(-i \nabla-\vec{A})^{2}$ in $L^{2}(M)$. Here again, to be more rigorous, $H_{A B}$ is the Friedrichs extension of the positive operator $(-i \nabla-\vec{A})^{2}$ defined on test functions on $M$.

For our purposes it would be more convenient to pass to a unitarily equivalent formulation. This is done in two steps. First, the differential operator $(-i \nabla-\vec{A})^{2}$ is lifted to $\tilde{M}$. Then the unitarily equivalent operator is $\tilde{H}_{A B}$ acting as a differential operator $(-i \nabla-\overrightarrow{\widetilde{A}})^{2}$ in the Hilbert space of $\Gamma$-periodic functions on $\tilde{M}$ which are square integrable over a fundamental domain of the $\Gamma$ action. Once more, $\tilde{H}_{A B}$ is rigorously introduced with the aid of the Friedrichs extension. Second, curl $\overrightarrow{\tilde{A}}=0$ holds again on $\tilde{M}$. But this time $\tilde{M}$ is simply connected and therefore the vector potential can be removed by a globally well defined gauge transformation. This gauge transformation induces a unitary mapping between the Hilbert space of $\Gamma$-periodic functions on $\tilde{M}$ and the Hilbert space $\mathscr{H}_{\Lambda}$ of $\Lambda$-equivariant functions on $\tilde{M}$, as introduced in Subsection 2.1. The resulting operator which is unitarily equivalent to $H_{A B}$ is nothing but the Hamiltonian $H_{\Lambda}=-\Delta$ acting in $\mathscr{H}_{\Lambda}$, as it has been introduced in the same subsection.

Remember that simultaneously one considers the free Hamiltonian $H=-\Delta$ in $L^{2}(\tilde{M})$. $H$ is $\Gamma$-periodic. In order to apply (5) and compute the propagator $\mathcal{K}^{\Lambda}(t, x, y)$ associated with $H_{\Lambda}$ one has to rely on a known formula for the free propagator $\mathcal{K}(t, x, y)$ on $\tilde{M}$.

Let us recall a formula for $\mathcal{K}(t, x, y)$, as presented in [21. Let $\vartheta$ stand for the Heaviside step function. For $x, y \in \tilde{M} \cup \mathcal{A} \cup \mathcal{B}$ set $\chi(x, y)=1$ if the points $x, y$ can be connected by a geodesic segment, and $\chi(x, y)=0$ otherwise. Given $t \in \mathbb{R}$ we define

$$
Z(t, x, y)=\vartheta(t) \chi(x, y) \frac{1}{4 \pi i t} \exp \left(\frac{i}{4 t} \operatorname{dist}^{2}(x, y)\right)
$$

furthermore, for $x_{1}, x_{3} \in \tilde{M} \cup \mathcal{A} \cup \mathcal{B}$ and $x_{2} \in \mathcal{A} \cup \mathcal{B}$ obeying $\chi\left(x_{1}, x_{2}\right)=\chi\left(x_{2}, x_{3}\right)=1$, and for $t_{1}, t_{2}>0$, we set

$$
V\left(\begin{array}{c}
x_{3}, x_{2}, x_{1} \\
t_{2}, t_{1}
\end{array}\right)=2 i\left(\left(\theta-\pi+i \ln \frac{t_{2} r_{1}}{t_{1} r_{2}}\right)^{-1}-\left(\theta+\pi+i \ln \frac{t_{2} r_{1}}{t_{1} r_{2}}\right)^{-1}\right)
$$

where $\theta=\angle x_{1}, x_{2}, x_{3} \in \mathbb{R}$ is the oriented angle and $r_{1}=\operatorname{dist}\left(x_{1}, x_{2}\right), r_{2}=\operatorname{dist}\left(x_{2}, x_{3}\right)$. Note that $\theta$ can take any real value.

We claim that the free propagator on $\tilde{M}$ equals

$$
\mathcal{K}\left(t, x, x_{0}\right)=\sum_{\gamma \in \mathscr{C}\left(x, x_{0}\right)} \mathcal{K}_{\gamma}\left(t, x, x_{0}\right)
$$

where $\mathscr{C}\left(x, x_{0}\right)$ stands for the set of all piecewise geodesic curves $\gamma: x_{0} \rightarrow C_{1} \rightarrow \cdots \rightarrow C_{n} \rightarrow x$ with the inner vortices $C_{j}, 1 \leq j \leq n$, belonging to the set of extreme points $\mathcal{A} \cup \mathcal{B}$. This means that it should hold $\chi\left(x_{0}, C_{1}\right)=\chi\left(C_{1}, C_{2}\right)=\cdots=\chi\left(C_{n}, x\right)=1$. Let us denote by $|\gamma|=n$ the length of the sequence $\left(C_{1}, C_{2}, \ldots, C_{n}\right)$. In particular, if $|\gamma|=0$ then $\gamma$ designates the geodesic segment $x_{0} \rightarrow x$. To simplify notation we set everywhere where convenient $C_{0}=x_{0}$ and $C_{n+1}=x$. With this convention, the terms in $(9)$ equal

$$
\mathcal{K}_{\gamma}\left(t, x, x_{0}\right)=\int_{\mathbb{R}^{n+1}} \mathrm{~d} t_{n} \cdots \mathrm{d} t_{0} \delta\left(t_{n}+\cdots+t_{0}-t\right) \prod_{j=0}^{n-1} V\left(\begin{array}{c}
C_{j+2}, C_{j+1}, C_{j} \\
t_{j+1}, t_{j}
\end{array}\right) \prod_{j=0}^{n} Z\left(t_{j}, C_{j+1}, C_{j}\right) .
$$

In particular, if $|\gamma|=0$ then $\mathcal{K}_{\gamma}\left(t, x, x_{0}\right)=Z\left(t, x, x_{0}\right)$, and if $|\gamma|=1$ then $\gamma$ designates a path composed of two geodesic segments $x_{0} \rightarrow C \rightarrow x$, with $C \in \mathcal{A} \cup \mathcal{B}$, and

$$
\mathcal{K}_{\gamma}\left(t, x, x_{0}\right)=\vartheta(t) \int_{0}^{t} V\left(\begin{array}{c}
x, C, x_{0} \\
t-s, s
\end{array}\right) Z(t-s, x, C) Z\left(s, C, x_{0}\right) \mathrm{d} s .
$$

In what follows we aim to provide a detailed verification of formulas $(9), 10$. 


\subsection{Auxiliary Relations}

In $\mathbb{R}^{2}$, it holds true that

$$
\left(\frac{\partial}{\partial x}+i \frac{\partial}{\partial y}\right) \frac{1}{x+i y}=2 \pi \delta(x) \delta(y) \quad \text { and } \quad \Delta \frac{1}{x+i y}=2 \pi\left(\delta(y) \delta^{\prime}(x)-i \delta(x) \delta^{\prime}(y)\right) .
$$

It follows that

$$
\left(\frac{\partial^{2}}{\partial r^{2}}+\frac{1}{r} \frac{\partial}{\partial r}+\frac{1}{r^{2}} \frac{\partial^{2}}{\partial \theta^{2}}\right)\left(\theta+i \ln \frac{t}{r}\right)^{-1}=\frac{2 \pi t}{r^{2}}\left(\delta(t-r) \delta^{\prime}(\theta)-i r \delta^{\prime}(t-r) \delta(\theta)\right)
$$

holds on the domain $t>0, r>0, \theta \in \mathbb{R}$. On the same domain,

$$
\left(r \frac{\partial}{\partial r}+t \frac{\partial}{\partial t}\right)\left(\theta+i \ln \frac{t}{r}\right)^{-1}=0 .
$$

Combining (11) and 12 one finds that

$$
\begin{aligned}
\left(i \frac{\partial}{\partial t}+\frac{\partial^{2}}{\partial r^{2}}+\frac{1}{r} \frac{\partial}{\partial r}+\frac{1}{r^{2}} \frac{\partial^{2}}{\partial \theta^{2}}\right)\left(\theta+i \ln \frac{t}{r}\right)^{-1} \frac{1}{t} \exp \left(i \frac{r^{2}}{4 t}\right) & \\
& =\frac{2 \pi}{r^{2}} \exp \left(i \frac{r^{2}}{4 t}\right)\left(\delta(t-r) \delta^{\prime}(\theta)-i r \delta^{\prime}(t-r) \delta(\theta)\right)
\end{aligned}
$$

Equipped with (13) one can prove the identity

$$
\begin{aligned}
\left(i \frac{\partial}{\partial t}+\frac{\partial^{2}}{\partial r^{2}}+\frac{1}{r} \frac{\partial}{\partial r}+\frac{1}{r^{2}} \frac{\partial^{2}}{\partial \theta^{2}}\right) \int_{0}^{t}\left(\theta+i \ln \frac{(t-s) r_{0}}{s r}\right)^{-1} \frac{1}{t-s} \exp \left(i \frac{r^{2}}{4(t-s)}\right) f(s) \mathrm{d} s & \\
=\frac{2 \pi r_{0}}{r^{2}\left(r+r_{0}\right)} \exp \left(i \frac{\left(r+r_{0}\right) r}{4 t}\right) & {\left[f\left(\frac{t r_{0}}{r+r_{0}}\right) \delta^{\prime}(\theta)\right.} \\
& \left.\quad-i \frac{r}{r+r_{0}}\left(\left(1+i \frac{r_{0}\left(r+r_{0}\right)}{4 t}\right) f\left(\frac{t r_{0}}{r+r_{0}}\right)+\frac{t r_{0}}{r+r_{0}} f^{\prime}\left(\frac{t r_{0}}{r+r_{0}}\right)\right) \delta(\theta)\right]
\end{aligned}
$$

which is true in the sense of distributions for any $r_{0}>0$ and $f \in C^{1}([0,+\infty[)$, again on the domain $t>0, r>0$, $\theta \in \mathbb{R}$. Note that

$$
\frac{1}{\varepsilon} \exp \left(i \frac{r^{2}}{4 \varepsilon}\right) \rightarrow 0 \text { as } \varepsilon \rightarrow 0+\text { in } \mathscr{D}^{\prime}(] 0,+\infty[) \text {. }
$$

In particular, letting $f(s)=(1 / s) \exp \left(i r_{0}^{2} /(4 s)\right)$ one derives the identity

$$
\begin{aligned}
\left(i \frac{\partial}{\partial t}+\frac{\partial^{2}}{\partial r^{2}}+\frac{1}{r} \frac{\partial}{\partial r}+\frac{1}{r^{2}} \frac{\partial^{2}}{\partial \theta^{2}}\right) \int_{0}^{t}\left(\theta+i \ln \frac{(t-s) r_{0}}{s r}\right)^{-1} \frac{1}{(t-s) s} \exp \left(i\left(\frac{r^{2}}{4(t-s)}+\frac{r_{0}^{2}}{4 s}\right)\right) \mathrm{d} s \\
=\frac{2 \pi}{t r^{2}} \exp \left(i \frac{\left(r+r_{0}\right)^{2}}{4 t}\right) \delta^{\prime}(\theta) .
\end{aligned}
$$

Let us further recall a basic fact concerning the generalized Laplacian. If $G \subset \tilde{M}$ is an open set with a piecewise smooth boundary, $\chi_{G}$ is the characteristic function of $G, \vec{n}$ is the normalized outer normal vector field on $\partial G$ and $\eta$ is a smooth function on $\tilde{M}$ then, in the sense of distributions,

$$
\Delta\left(\eta \chi_{G}\right)=(\Delta \eta) \chi_{G}-\frac{\partial \eta}{\partial \vec{n}} \delta_{\partial G}-\frac{\partial}{\partial \vec{n}}\left(\eta \delta_{\partial G}\right)
$$

The distribution $\delta_{\partial G}$ is a single layer supported on the curve $\partial G$ and fulfilling

$$
\forall \varphi \in C_{0}^{\infty}(\tilde{M}), \delta_{\partial G}(\varphi)=\int_{\partial G} \varphi \mathrm{d} \ell
$$

The double layer $\partial / \partial \vec{n}\left(\eta \delta_{\partial G}\right)$ is defined by

$$
\forall \varphi \in C_{0}^{\infty}(\tilde{M}),\left(\frac{\partial}{\partial \vec{n}}\left(\eta \delta_{\partial G}\right)\right)(\varphi)=-\int_{\partial G} \frac{\partial \varphi}{\partial \vec{n}} \mathrm{~d} \ell .
$$




\subsection{VERIFICATION OF THE PROPAGATOR FORMULA}

We have to show that, for $x_{0} \in \tilde{M}$ fixed, the propagator $\mathcal{K}\left(t, x, x_{0}\right)$ defined in $[9,, 10$, verifies the condition

$$
\left(i \frac{\partial}{\partial t}+\Delta\right) \mathcal{K}\left(t, x, x_{0}\right)=i \delta(t) \delta\left(x, x_{0}\right) \text { on } \mathbb{R} \times \tilde{M}
$$

This is equivalent to showing that

$$
\lim _{t \rightarrow 0_{+}} \mathcal{K}\left(t, x, x_{0}\right)=\delta\left(x, x_{0}\right)
$$

and

$$
\left(i \frac{\partial}{\partial t}+\Delta\right) \mathcal{K}\left(t, x, x_{0}\right)=0 \text { for } t>0, x \in \tilde{M}
$$

Equation 18 is obvious. In fact, since the form of $Z\left(t, x, x_{0}\right)$ on the sheet $\left\{x ; \chi\left(x, x_{0}\right)=1\right\}$ is that of the free propagator on $\mathbb{R}^{2}$ we have

$$
\lim _{t \rightarrow 0_{+}} Z\left(t, x, x_{0}\right)=\delta\left(x, x_{0}\right)
$$

By a similar reasoning, $\lim _{t \rightarrow 0_{+}} Z(t, x, C)=0$ if $C \in \mathcal{A} \cup \mathcal{B}$ and $x$ runs over $\tilde{M}$. Hence

$$
\lim _{t \rightarrow 0_{+}} \mathcal{K}_{\gamma}\left(t, x, x_{0}\right)=0 \text { if }|\gamma| \geq 1
$$

Concerning (19), we first introduce some notation related to the geometry of the universal covering space. Denote by $\varrho$ the distance $\operatorname{dist}(a, b)$. Observe that if $C_{1}, C_{2} \in \mathcal{A} \cup \mathcal{B}$ then $\chi\left(C_{1}, C_{2}\right)=1$ if and only if $\operatorname{dist}\left(C_{1}, C_{2}\right)=\varrho$. If this is the case then necessarily $C_{1} \in \mathcal{A}$ and $C_{2} \in \mathcal{B}$ or vice versa.

For $x \in \tilde{M} \cup \mathcal{A} \cup \mathcal{B}$ set

$$
D(x)=\{y \in \tilde{M} ; \chi(x, y)=1\} .
$$

If $x \in \tilde{M}$ then $D(x)$ can be identified with the plane cut along two half-lines with the limit points $a$ and $b$, respectively. The border of $D(x)$ consists of two pairs of half-lines. One pair has a common limit point $A \in \mathcal{A}$ and is denoted $\partial D(x ; A)$, the other pair has a common limit point $B \in \mathcal{B}$ and is denoted $\partial D(x ; B)$. We have

$$
\partial D(x)=\partial D(x ; A) \cup \partial D(x ; B) .
$$

If $C \in \mathcal{A} \cup \mathcal{B}$ then $D(C)$ resembles the universal covering space in the one-vortex case. It can be viewed as a union of countably many sheets glued together in a staircase-like way. Each sheet contributes to the border of $D(C)$ by a pair of half-lines with a common limit point $C^{\prime}$. Thus the border $\partial D(C)$ is formed by a countable union of pairs of half-lines:

$$
\partial D(C)=\bigcup_{C^{\prime} \in \mathcal{D}, \operatorname{dist}\left(C, C^{\prime}\right)=\varrho} \partial D\left(C ; C^{\prime}\right),
$$

where $\mathcal{D}=\mathcal{B}$ if $C \in \mathcal{A}$ and $\mathcal{D}=\mathcal{A}$ if $C \in \mathcal{B}$.

Let us first examine the case $|\gamma|=0$. One has

$$
\left(i \frac{\partial}{\partial t}+\Delta\right) Z\left(t, x, x_{0}\right)=0
$$

for $t>0$ and $x \in D\left(x_{0}\right)$. Observe also that

$$
\frac{\partial}{\partial \vec{n}} Z\left(t, x, x_{0}\right)=0 \text { for } x \in \partial D\left(x_{0}\right)
$$

This is so since, in polar coordinates centered at $x_{0}, Z\left(t, x, x_{0}\right)$ does not depend on the angle variable. Let us also note that $Z\left(t, x, x_{0}\right)$ can be continued smoothly in the variable $x$ over the borderline of the domain $D\left(x_{0}\right)$. Thus, in virtue of 16 , for $t>0$ and $x \in \tilde{M}$,

$$
\left(i \frac{\partial}{\partial t}+\Delta\right) Z\left(t, x, x_{0}\right)=-\frac{\partial}{\partial \vec{n}}\left(Z\left(t, x, x_{0}\right) \delta_{\partial D\left(x_{0}\right)}\right)
$$

Remark. In (23) as well as everywhere in this section we use the following convention. The value of a density (which is in this case $Z\left(t, x, x_{0}\right)$ ) on the border $\partial D\left(x_{0}\right)$ is understood as the limit value taken from the interior of the domain $D\left(x_{0}\right)$. 
Next we discuss the case $|\gamma|=1$. Then $\gamma$ designates a piecewise geodesic curve $x_{0} \rightarrow C \rightarrow x$, with $C \in \mathcal{A} \cup \mathcal{B}$. Denote by $\gamma^{\prime}$ the geodesic segment $x_{0} \rightarrow x$ provided $x \in D\left(x_{0}\right)$. We have

$$
\begin{aligned}
& \mathcal{K}_{\gamma}\left(t, x, x_{0}\right)=\frac{1}{8 \pi^{2} i} \chi(x, C) \chi\left(C, x_{0}\right) \\
& \quad \times \int_{0}^{t}\left(\left(\theta-\pi+i \ln \frac{(t-s) r_{0}}{s r}\right)^{-1}-\left(\theta+\pi+i \ln \frac{(t-s) r_{0}}{s r}\right)^{-1}\right) \frac{1}{(t-s) s} \exp \left(i\left(\frac{r^{2}}{4(t-s)}+\frac{r_{0}^{2}}{4 s}\right)\right) \mathrm{d} s
\end{aligned}
$$

where $r=\operatorname{dist}(x, C), r_{0}=\operatorname{dist}\left(C, x_{0}\right)$ and $\theta=\angle x_{0}, C, x$.

Application of the differential operator $\left(i \partial_{t}+\Delta\right)$ to the RHS of $(24)$ in the sense of distributions results in several singular terms supported on one-dimensional submanifolds. First, due to the discontinuity of the characteristic function $\chi(x, C)$, the application of $\Delta$ leads to two terms supported on the boundary $\partial D(C)$ (see (16)). Second, as it follows from (15), the singularity of the integrand for the values $\theta= \pm \pi$ and $r_{0} / s=r /(t-s)$ produces terms supported on the submanifold determined by $\theta= \pm \pi$, and this set is nothing but a part of the boundary of the domain $D\left(x_{0}\right)$, namely $\partial D\left(x_{0} ; C\right)$. Notice that for $\theta= \pm \pi$ it holds $r+r_{0}=\operatorname{dist}\left(x, x_{0}\right)$ and $\partial / \partial \vec{n}= \pm r^{-1} \partial / \partial \theta$. Moreover, in polar coordinates centered at $C$,

$$
\delta_{\partial D\left(x_{0} ; C\right)}=\frac{1}{r}(\delta(\theta-\pi)+\delta(\theta+\pi)) .
$$

Thus the latter contribution takes the form

$$
\frac{1}{4 \pi i t r^{2}} \frac{\partial}{\partial \theta}\left(\exp \left(\frac{i}{4 t} \operatorname{dist}\left(x, x_{0}\right)^{2}\right)(\delta(\theta-\pi)-\delta(\theta+\pi))\right)=\frac{\partial}{\partial \vec{n}}\left(\mathcal{K}_{\gamma^{\prime}}\left(t, x, x_{0}\right) \delta_{\partial D\left(x_{0}, C\right)}\right),
$$

where $\mathcal{K}_{\gamma^{\prime}}\left(t, x, x_{0}\right)=Z\left(t, x, x_{0}\right)$. In summary, we obtain

$$
\begin{aligned}
\left(i \frac{\partial}{\partial t}+\Delta\right) \mathcal{K}_{\gamma}\left(t, x, x_{0}\right)=-\left(\frac{\partial}{\partial \vec{n}} \mathcal{K}_{\gamma}\left(t, x, x_{0}\right)\right) & \delta_{\partial D(C)} \\
& -\frac{\partial}{\partial \vec{n}}\left(\mathcal{K}_{\gamma}\left(t, x, x_{0}\right) \delta_{\partial D(C)}\right)+\frac{\partial}{\partial \vec{n}}\left(\mathcal{K}_{\gamma^{\prime}}\left(t, x, x_{0}\right) \delta_{\partial D\left(x_{0}, C\right)}\right)
\end{aligned}
$$

Finally, let us consider the case $|\gamma| \geq 2$. Thus $\gamma$ is a piecewise geodesic curve $x_{0} \rightarrow C_{1} \rightarrow \cdots \rightarrow C_{n} \rightarrow x$, $n \geq 2$. Denote by $\gamma^{\prime}$ the truncated geodesic curve $x_{0} \rightarrow C_{1} \rightarrow \cdots \rightarrow C_{n-1} \rightarrow x$ provided $x \in D\left(C_{n-1}\right)$. Recalling (7), (8), one can express

$$
\begin{aligned}
& \mathcal{K}_{\gamma}\left(t, x, x_{0}\right)=\int_{\mathbb{R}^{n}} \mathrm{~d} t_{n-1} \ldots \mathrm{d} t_{0} V\left(\begin{array}{c}
x, C_{n}, C_{n-1} \\
t-\tau, t_{n-1}
\end{array}\right) Z\left(t-\tau, x, C_{n}\right) F_{\gamma}\left(t_{0}, \ldots, t_{n-1}, x_{0}\right) \\
& =\frac{1}{2 \pi} \chi\left(x, C_{n}\right) \int_{\mathbb{R}^{n-1}} \mathrm{~d} t_{n-2} \ldots \mathrm{d} t_{0} \int_{0}^{t-\tau^{\prime}} \mathrm{d} t_{n-1}\left(\left(\theta-\pi+i \ln \frac{\left(t-\tau^{\prime}-t_{n-1}\right) \varrho}{t_{n-1} r}\right)^{-1}\right. \\
& \left.\quad-\left(\theta+\pi+i \ln \frac{\left(t-\tau^{\prime}-t_{n-1}\right) \varrho}{t_{n-1} r}\right)^{-1}\right) \frac{1}{t-\tau^{\prime}-t_{n-1}} \exp \left(i \frac{r^{2}}{4\left(t-\tau^{\prime}-t_{n-1}\right)}\right) F_{\gamma}\left(t_{0}, \ldots, t_{n-1}, x_{0}\right)
\end{aligned}
$$

where

$$
\tau=t_{0}+\cdots+t_{n-2}+t_{n-1}, \quad \tau^{\prime}=t_{0}+\cdots+t_{n-2}, \quad r=\operatorname{dist}\left(C_{n}, x\right), \quad \theta=\angle C_{n-1}, C_{n}, x,
$$

and

$$
F_{\gamma}\left(t_{0}, \ldots, t_{n-1}, x_{0}\right)=\prod_{j=0}^{n-2} V\left(\begin{array}{c}
C_{j+2}, C_{j+1}, C_{j} \\
t_{j+1}, t_{j}
\end{array}\right) \prod_{j=0}^{n-1} Z_{t_{j}}\left(C_{j+1}, C_{j}\right) .
$$

Application of $\left(i \partial_{t}+\Delta\right)$ to the RHS of 26 in the sense of distributions again produces several singular terms. As a consequence of the discontinuity of the characteristic function $\chi\left(x, C_{n}\right)$ a single and a double layer supported on the boundary $\partial D\left(C_{n}\right)$ occur (see $(16)$ ). The singularity of the integrand for the values $\theta= \pm \pi$ and $\varrho / t_{n-1}=r /\left(t-\tau^{\prime}-t_{n-1}\right)$ produces terms supported on the part of the boundary of the domain $D\left(C_{n-1}\right)$, namely on $\partial D\left(C_{n-1} ; C_{n}\right)$. This time one can apply identity 14 .

In order to treat the resulting terms the following equations are useful. Suppose that $\theta= \pm \pi$ and so $x \in \partial D\left(C_{n-1} ; C_{n}\right)$. Set

$$
r^{\prime}=r+\varrho=\operatorname{dist}\left(C_{n-1}, x\right), \quad \theta^{\prime}=\angle C_{n-2}, C_{n-1}, x .
$$

If $\varrho / t_{n-1}=r /\left(t-\tau^{\prime}-t_{n-1}\right)$ then

$$
t_{n-1}=\frac{\varrho\left(t-\tau^{\prime}\right)}{r^{\prime}} \text { and } \quad \frac{t-\tau^{\prime}-t_{n-1}}{r}=\frac{t-\tau^{\prime}}{r^{\prime}} .
$$


Moreover,

$$
\frac{\varrho}{r^{\prime}} \exp \left(\frac{i r^{2}}{4(t-\tau)}\right) Z\left(t_{n-1}, C_{n}, C_{n-1}\right)=Z\left(t-\tau^{\prime}, x, C_{n-1}\right)
$$

and

$$
V\left(\begin{array}{c}
C_{n}, C_{n-1}, C_{n-2} \\
\varrho s_{2} / r^{\prime}, s_{1}
\end{array}\right)=V\left(\begin{array}{c}
x, C_{n-1}, C_{n-2} \\
s_{2}, s_{1}
\end{array}\right)
$$

Observe also that

$$
\begin{gathered}
\left.\frac{\partial}{\partial s}\left(\exp \left(\frac{i r^{2}}{4\left(t-\tau^{\prime}-s\right)}\right) \exp \left(\frac{i \varrho^{2}}{4 s}\right)\right)\right|_{s=\varrho\left(t-\tau^{\prime}\right) / r^{\prime}}=0, \\
\left.\exp \left(\frac{i r^{2}}{4\left(t-\tau^{\prime}-s\right)}\right) \exp \left(\frac{i \varrho^{2}}{4 s}\right)\right|_{s=\varrho\left(t-\tau^{\prime}\right) / r^{\prime}}=\exp \left(\frac{i r^{\prime 2}}{4\left(t-\tau^{\prime}\right)}\right),
\end{gathered}
$$

and for $\theta=\pi$,

$$
\left.\frac{\partial}{\partial s} V\left(\begin{array}{c}
C_{n}, C_{n-1}, C_{n-2} \\
s, t_{n-2}
\end{array}\right)\right|_{s=\varrho\left(t-\tau^{\prime}\right) / r^{\prime}}=\frac{i r^{\prime}}{\varrho\left(t-\tau^{\prime}\right)} \frac{\partial}{\partial \theta^{\prime}} V\left(\begin{array}{c}
x, C_{n-1}, C_{n-2} \\
t-\tau^{\prime}, t_{n-2}
\end{array}\right) .
$$

A similar relation holds for $\theta=-\pi$.

After a bit tedious but quite straightforward manipulations one arrives at the final identity

$$
\begin{aligned}
\left(i \frac{\partial}{\partial t}+\Delta\right) \mathcal{K}_{\gamma}\left(t, x, x_{0}\right)=-\left(\frac{\partial}{\partial \vec{n}} \mathcal{K}_{\gamma}\left(t, x, x_{0}\right)\right) \delta_{\partial D\left(C_{n}\right)}-\frac{\partial}{\partial \vec{n}}\left(\mathcal{K}_{\gamma}\left(t, x, x_{0}\right) \delta_{\partial D\left(C_{n}\right)}\right) \\
+\left(\frac{\partial}{\partial \vec{n}} \mathcal{K}_{\gamma^{\prime}}\left(t, x, x_{0}\right)\right) \delta_{\partial D\left(C_{n-1} ; C_{n}\right)}+\frac{\partial}{\partial \vec{n}}\left(\mathcal{K}_{\gamma^{\prime}}\left(t, x, x_{0}\right) \delta_{\partial D\left(C_{n-1} ; C_{n}\right)}\right)
\end{aligned}
$$

Now we can show $(19)$ when taking into account 23,25 and 27 . It is true that

$$
\begin{aligned}
&\left(i \frac{\partial}{\partial t}+\Delta\right) \mathcal{K}\left(t, x, x_{0}\right)=\sum_{|\gamma| \geq 2}[--\left(\frac{\partial}{\partial \vec{n}} \mathcal{K}_{\gamma}\left(t, x, x_{0}\right)\right) \delta_{\partial D\left(C_{n}\right)}-\frac{\partial}{\partial \vec{n}}\left(\mathcal{K}_{\gamma}\left(t, x, x_{0}\right) \delta_{\partial D\left(C_{n}\right)}\right) \\
&\left.+\left(\frac{\partial}{\partial \vec{n}} \mathcal{K}_{\gamma^{\prime}}\left(t, x, x_{0}\right)\right) \delta_{\partial D\left(C_{n-1} ; C_{n}\right)}+\frac{\partial}{\partial \vec{n}}\left(\mathcal{K}_{\gamma^{\prime}}\left(t, x, x_{0}\right) \delta_{\partial D\left(C_{n-1} ; C_{n}\right)}\right)\right] \\
&+\sum_{|\gamma|=1}\left[-\left(\frac{\partial}{\partial \vec{n}} \mathcal{K}_{\gamma}\left(t, x, x_{0}\right)\right) \delta_{\partial D(C)}-\frac{\partial}{\partial \vec{n}}\left(\mathcal{K}_{\gamma}\left(t, x, x_{0}\right) \delta_{\partial D(C)}\right)\right. \\
&\left.+\frac{\partial \vec{r}}{\partial \vec{n}}\left(Z\left(t, x, x_{0}\right) \delta_{\partial D\left(x_{0} ; C\right)}\right)\right]-\frac{\partial}{\partial \vec{n}}\left(Z\left(t, x, x_{0}\right) \delta_{\partial D\left(x_{0}\right)}\right)=0,
\end{aligned}
$$

where we have used 21 and 22 .

\section{Conclusion. The propagator for two Aharonov-Bohm vortices}

In conclusion we present a formula for the propagator of a charged particle on the plane pierced by two Aharonov-Bohm magnetic fluxes.

Without loss of generality we can suppose that the vortices are located at the points $a=(0,0)$ and $b=(\varrho, 0)$. In order to express the propagator for the Aharonov-Bohm Hamiltonian $H_{A B}$ we again pass to a unitarily equivalent formulation. Let us cut the plane along two half-lines,

$$
\left.L_{a}=\right]-\infty, 0\left[\times\{0\} \text { and } L_{b}=\right] \varrho,+\infty[\times\{0\}
$$

Let $\left(r_{a}, \theta_{a}\right)$ be polar coordinates centered at the point $a$ and $\left(r_{b}, \theta_{b}\right)$ be polar coordinates centered at the point $b$. The angle variables are chosen so that the values $\theta_{a}= \pm \pi$ correspond to the two sides of the cut $L_{a}$, and similarly for $\theta_{b}$ and $L_{b}$. Then an explicit and commonly used choice of the Aharonov-Bohm vector potential reads

$$
\vec{A}=\alpha \nabla \theta_{a}+\beta \nabla \theta_{b}
$$

Denote by $U$ a unitary operator in $L^{2}\left(\mathbb{R}^{2}, \mathrm{~d}^{2} x\right)$ acting as the multiplication operator

$$
U \psi=e^{i\left(\alpha \theta_{a}+\beta \theta_{b}\right)} \psi
$$


and let $H_{\Lambda}^{\prime}=U^{-1} H_{A B} U$. Then $H_{\Lambda}^{\prime}$ acts as $-\Delta$ in $L^{2}\left(\mathbb{R}^{2}, \mathrm{~d}^{2} x\right)$, and its domain is determined by the boundary conditions along the cut $L_{a} \cup L_{b}$ :

$$
\begin{array}{ll}
\psi\left(r_{a}, \theta_{a}=\pi\right)=e^{2 \pi i \alpha} \psi\left(r_{a}, \theta_{a}=-\pi\right), & \partial_{\theta_{a}} \psi\left(r_{a}, \theta_{a}=\pi\right)=e^{2 \pi i \alpha} \partial_{\theta_{a}} \psi\left(r_{a}, \theta_{a}=-\pi\right), \\
\psi\left(r_{b}, \theta_{b}=\pi\right)=e^{2 \pi i \beta} \psi\left(r_{b}, \theta_{b}=-\pi\right), & \partial_{\theta_{b}} \psi\left(r_{b}, \theta_{b}=\pi\right)=e^{2 \pi i \beta} \partial_{\theta_{b}} \psi\left(r_{b}, \theta_{b}=-\pi\right) .
\end{array}
$$

In addition, one imposes the regular boundary condition at the vortices, namely $\psi(a)=\psi(b)=0$.

We wish to find a formula for the propagator $\mathcal{K}_{A B}\left(t, x, x_{0}\right)$ associated with the Hamiltonian $H_{A B}$. Note that

$$
\mathcal{K}_{A B}\left(t, x, x_{0}\right)=\exp ^{i\left(\alpha \theta_{a}(x)+\beta \theta_{b}(x)\right)} \mathcal{K}^{\prime \Lambda}\left(t, x, x_{0}\right) e^{-i\left(\alpha \theta_{a}\left(x_{0}\right)+\beta \theta_{b}\left(x_{0}\right)\right)}
$$

where $\mathcal{K}^{\prime \Lambda}\left(t, x, x_{0}\right)$ is the propagator associated with $H^{\prime}{ }_{\Lambda}$. Let us denote

$$
D=\mathbb{R}^{2} \backslash\left(L_{a} \cup L_{b}\right) .
$$

Then one can embed $D \subset \tilde{M}$ as a fundamental domain. $\mathcal{K}^{\prime \Lambda}\left(t, x, x_{0}\right)$ is simply obtained as the restriction to $D$ of the propagator $\mathcal{K}^{\Lambda}\left(t, x, x_{0}\right)$ associated with the Hamiltonian $H_{\Lambda}$. On the other hand, to construct $\mathcal{K}^{\Lambda}\left(t, x, x_{0}\right)$ one can apply formula (5) and the knowledge of the free propagator on $\tilde{M}$, see (9), (10). Thus we get

$$
\mathcal{K}^{\Lambda}\left(t, x, x_{0}\right)=\sum_{g \in \Gamma} \sum_{\gamma \in \mathscr{C}\left(g \cdot x, x_{0}\right)} \Lambda\left(g^{-1}\right) \mathcal{K}_{\gamma}\left(t, g \cdot x, x_{0}\right)
$$

Fix $t>0$ and $x_{0}, x \in D$. One can classify piecewise geodesic paths in $\tilde{M}$,

$$
\gamma: x_{0} \rightarrow C_{1} \rightarrow \cdots \rightarrow C_{n} \rightarrow g \cdot x,
$$

with $C_{j} \in \mathcal{A} \cup \mathcal{B}$ and $g \in \Gamma$, according to their projections to $M$. Let $\bar{\gamma}$ be a finite alternating sequence of points $a$ and $b$, i.e. $\bar{\gamma}=\left(c_{1}, \ldots, c_{n}\right), c_{j} \in\{a, b\}$ and $c_{j} \neq c_{j+1}$. The empty sequence $\bar{\gamma}=()$ is admissible. Relate to $\bar{\gamma}$ a piecewise geodesic path in $M$, namely $x_{0} \rightarrow c_{1} \rightarrow \cdots \rightarrow c_{n} \rightarrow x$. Suppose that this path is covered by a path $\gamma$ in $\tilde{M}$, as given in 29. Then $C_{j} \in \mathcal{A}$ iff $c_{j}=a$ and $C_{j} \in \mathcal{B}$ iff $c_{j}=b$. Denote the angles $\angle x_{0}, c_{1}, c_{2}=\theta_{0}$ and $\angle c_{n-1}, c_{n}, x=\theta$. Then the angles in the path $\gamma$ in $(29)$ take the values $\angle x_{0}, C_{1}, C_{2}=\theta_{0}+2 \pi k_{1}, \angle C_{n-1}, C_{n}, g \cdot x=\theta+2 \pi k_{n}$ and $\angle C_{j}, C_{j+1}, C_{j+2}=2 \pi k_{j+1}$ for $1 \leq j \leq n-2$ (if $n \geq 3$ ), where $k_{1}, \ldots, k_{n}$ are integers. Any values $k_{1}, \ldots, k_{n} \in \mathbb{Z}$ are possible. In that case the representation $\Lambda$ applied to the group element $g$ occurring in $(29)$ takes the value

$$
\Lambda(g)=\exp \left(2 \pi i\left(k_{1} \sigma_{1}+\cdots+k_{n} \sigma_{n}\right)\right)
$$

where $\sigma_{j} \in\{\alpha, \beta\}$ and $\sigma_{j}=\alpha$ if $c_{j}=a$, and $\sigma_{j}=\beta$ if $c_{j}=b$.

Using the equation

$$
\sum_{k \in \mathbb{Z}} e^{-2 \pi \mathrm{i} \alpha k}\left(\frac{1}{\theta+2 \pi k-\pi+\mathrm{i} s}-\frac{1}{\theta+2 \pi k+\pi+\mathrm{i} s}\right)=-2 \sin (\pi \alpha) \frac{e^{-\alpha(s-\mathrm{i} \theta)}}{1+e^{-s+\mathrm{i} \theta}}
$$

which is valid for $0<\alpha<1,|\theta|<\pi$, one can carry out a partial summation in 28 over the integers $k_{1}, \ldots, k_{n}$. This way the double sum in 28 reduces to a sum over finite alternating sequences $\bar{\gamma}$.

Here is the resulting formula for $\mathcal{K}^{\prime \Lambda}\left(t, x, x_{0}\right)$. We set

$$
\zeta_{a}=1 \quad \text { or } \quad \zeta_{a}=e^{2 \pi i \alpha} \quad \text { or } \quad \zeta_{a}=e^{-2 \pi i \alpha}
$$

depending on whether the segment $\overline{x_{0} x}$ does not intersect $L_{a}$, or $\overline{x_{0} x}$ intersects $L_{a}$ and $x_{0}$ lies in the lower half-plane, or $\overline{x_{0} x}$ intersects $L_{a}$ and $x_{0}$ lies in the upper half-plane. Analogously,

$$
\zeta_{b}=1 \quad \text { or } \quad \zeta_{b}=e^{2 \pi i \beta} \quad \text { or } \quad \zeta_{b}=e^{-2 \pi i \beta}
$$

depending on whether the segment $\overline{x_{0} x}$ does not intersect $L_{b}$, or $\overline{x_{0} x}$ intersects $L_{b}$ and $x_{0}$ lies in the upper half-plane, or $\overline{x_{0} x}$ intersects $L_{b}$ and $x_{0}$ lies in the lower half-plane. Furthermore, let us write

$$
\zeta_{a}=e^{i \alpha \eta_{a}}, \quad \zeta_{b}=e^{i \beta \eta_{b}}, \quad \text { where } \eta_{a}, \eta_{b} \in\{0,2 \pi,-2 \pi\} .
$$

Then one has

$$
\begin{aligned}
& \mathcal{K}^{\prime \Lambda}\left(t, x, x_{0}\right)=\zeta_{a} \zeta_{b} \frac{1}{4 \pi i t} \exp \left(i \frac{\left|x-x_{0}\right|^{2}}{4 t}\right) \\
& \quad-\sum_{c \in\{a, b\}} \zeta_{c} \frac{\sin (\pi \sigma)}{4 \pi^{2} i} \int_{0}^{\infty} \frac{\mathrm{d} t_{1}}{t_{1}} \int_{0}^{\infty} \frac{\mathrm{d} t_{0}}{t_{0}} \delta\left(t_{1}+t_{0}-t\right) \exp \left(i\left(\frac{r_{c}^{2}}{4 t_{1}}+\frac{r_{0 c}^{2}}{4 t_{0}}\right)\right) \frac{\exp \left[-\sigma\left(s_{c}-i\left(\theta_{c}-\theta_{0 c}-\eta_{c}\right)\right]\right.}{1+\exp \left(-s_{c}+i \theta_{c}-i \theta_{0 c}\right)} \\
& +\frac{1}{4 \pi i} \sum_{\bar{\gamma}, n \geq 2}(-1)^{n} \int_{0}^{\infty} \frac{\mathrm{d} t_{n}}{t_{n}} \cdots \int_{0}^{\infty} \frac{\mathrm{d} t_{0}}{t_{0}} \delta\left(t_{n}+\cdots+t_{0}-t\right) \exp \left(\frac{i}{4}\left(\frac{r^{2}}{t_{n}}+\frac{\varrho^{2}}{t_{n-1}}+\cdots+\frac{\varrho^{2}}{t_{1}}+\frac{r_{0}^{2}}{t_{0}}\right)\right) S \bar{\gamma}\left(s, \theta, \theta_{0}\right),
\end{aligned}
$$


where

$$
S_{\bar{\gamma}}\left(s, \theta, \theta_{0}\right)=\frac{\sin \left(\pi \sigma_{n}\right)}{\pi} \frac{\exp \left[-\sigma_{n}\left(s_{n}-i \theta\right)\right]}{1+\exp \left(-s_{n}+i \theta\right)} \frac{\sin \left(\pi \sigma_{n-1}\right)}{\pi} \frac{\exp \left(-\sigma_{n-1} s_{n-1}\right)}{1+\exp \left(-s_{n-1}\right)} \times \cdots \times \frac{\sin \left(\pi \sigma_{1}\right)}{\pi} \frac{\exp \left[-\sigma_{1}\left(s_{1}-i \theta_{0}\right)\right]}{1+\exp \left(-s_{1}+i \theta_{0}\right)},
$$

and

$$
s_{a}=\ln \frac{t_{1} r_{0 a}}{t_{0} r_{a}}, \quad s_{b}=\ln \frac{t_{1} r_{0 b}}{t_{0} r_{b}}, \quad s_{j}=\ln \frac{t_{j} r_{j-1}}{t_{j-1} r_{j}} \quad \text { for } 1 \leq j \leq n .
$$

Furthermore, in the first sum on the RHS, $\left(r_{c}, \theta_{c}\right)$ and $\left(r_{0 c}, \theta_{0 c}\right)$ are the polar coordinates of $x$ and $x_{0}$ with respect to the center $c$, respectively, and $\sigma=\alpha$ (resp. $\beta$ ) if $c=a$ (resp. b). The second sum, $\sum_{\bar{\gamma}, n \geq 2}$, runs over all finite alternating sequences of length at least two, $\bar{\gamma}=\left(c_{1}, \ldots, c_{n}\right)$, and $(r, \theta)$ are the polar coordinates of $x$ with respect to $c_{n},\left(r_{0}, \theta_{0}\right)$ are the polar coordinates of $x_{0}$ with respect to $c_{1}$, and $\sigma_{j}=\alpha$ (resp. $\left.\beta\right)$ depending on whether $c_{j}=a$ (resp. $\left.b\right)$.

\section{ACKNOWLEDGEMENTS}

One of the authors (P.S. $)$ wishes to acknowledge gratefully partial support from grant GA13-11058S of the Czech Science Foundation.

\section{REFERENCES}

[1] I. Alexandrova, H. Tamura. Resonance free regions in magnetic scattering by two solenoidal fields at large separation. J. Funct. Anal. 260:1836-1885, 2011. DOI:10.1016/j.jfa.2010.12.005

[2] I. Alexandrova, H. Tamura. Resonances in scattering by two magnetic fields at large separation and a complex scaling method. Adv. Math. 256:398-448, 2014. DOI:10.1016/j.aim.2014.01.022

[3] J. Asch, H. Over, R. Seiler. Magnetic Bloch analysis and Bochner Laplacians. J. Geom. Phys. 13:275-288, 1994. DOI:10.1016/0393-0440(94)90035-3

[4] M. F. Atiyah. Elliptic operators, discrete groups and von Neumann algebras. Astérisque 32-33:43-72, 1976.

[5] O. Giraud, A. Thain, J. H. Hannay. Shrunk loop theorem for the topology probabilities of closed Brownian (or Feynman) paths on the twice punctured plane. J. Phys. A: Math. Gen. 37:2913-2935, 2004. DOI:10.1088/0305-4470/37/8/005

[6] M. J. Gruber. Bloch theory and quantization of magnetic systems. J. Geom. Phys. 34:137-154, 2000. DOI:10.1016/S0393-0440(99)00059-5

[7] J. H. Hannay, A. Thain. Exact scattering theory for any straight reflectors in two dimensions. J. Phys. A: Math. Gen. 36:4063-4080, 2003. DOI:10.1088/0305-4470/36/14/310

[8] L. Hörmander. The Analysis of Linear Partial Differential Operators I. Berlin: Springer, 2003.

[9] H. T. Ito, H. Tamura. Aharonov-Bohm effect in scattering by point-like magnetic fields at large separation. Ann. H. Poincaré 2:309-359, 2001. DOI:10.1007/PL00001036

[10] T. Kato: Perturbation theory of linear operators. New York: Springer-Verlag, 1966.

[11] P. Kocábová, P. Štovíček. Generalized Bloch analysis and propagators on Riemannian manifolds with a discrete symmetry. J. Math. Phys. 49:art. no. 033518, 2008. DOI:10.1063/1.2898484

[12] P. Koštáková, P. Štovíček. Noncommutative Bloch analysis of Bochner Laplacians with nonvanishing gauge fields. J. Geom. Phys. 61:727-744, 2011. DOI:10.1016/j.geomphys.2010.12.004

[13] J. M. Lee. Introduction to Topological Manifolds. Berlin: Springer-Verlag, 2000.

[14] S. Mashkevich, J. Myrheim, S. Ouvry. Quantum mechanics of a particle with two magnetic impurities. Phys. Lett. A 330:41-47, 2004. DOI:10.1016/j.physleta.2004.07.040

[15] M. Melgaard, E. Ouhabaz, G. Rozenblum. Negative discrete spectrum of perturbed multivortex Aharonov-Bohm Hamiltonians. Ann. H. Poincaré 5:979-1012, 2004. DOI:10.1007/s00023-004-0187-3

[16] T. Mine. Periodic Aharonov-Bohm solenoids in a constant magnetic field. Ann. H. Poincaré 6:125-154, 2005. DOI:10.1007/s00023-005-0201-4

[17] T. Mine, Y. Nomura. Periodic Aharonov-Bohm solenoids in a constant magnetic field. Rev. Math. Phys. 18:913-934, 2006. DOI:10.1142/S0129055X06002826

[18] J. S. Schulman. Approximate topologies. J. Math. Phys. 12:304-308, 1971. DOI:10.1063/1.1665592

[19] J. S. Schulman. Techniques and Applications of Path Integration. New York: Wiley, 1981.

[20] A. J. Shtern. Unitary representation of a topological group The Online Encyclopaedia of Mathematics. Berlin: Springer, 2001. Online: http://eom.springer.de/

[21] P. Štovíček. The Green function for the two-solenoid Aharonov-Bohm effect. Phys. Lett. A 142:5-10, 1989. DOI:10.1016/0375-9601(89)90702-0 
[22] P. Štovíček. Scattering on a finite chain of vortices. Duke Math. J. 76:303-332, 1994. DOI:10.1215/S0012-7094-94-07611-4

[23] T. Sunada. Fundamental groups and Laplacians. In: Geometry and analysis on manifolds. Lect. Notes Math. 1339. Berlin: Springer, 1988, pp. 248-277.

[24] T. Sunada. A periodic Schrödinger operator in an abelian cover, J. Fac. Sci. Univ. Tokyo Sect., 1A Math. 37:575-583, 1990.

[25] H. Tamura. Semiclassical analysis for magnetic scattering by two solenoidal fields: Total cross sections. Ann. H. Poincaré 8:1071-1114, 2007. DOI:10.1007/s00023-007-0329-5

[26] E. Thoma. Über unitäre Darstellungen abzälbarer, diskreter Gruppen. Math. Annalen 153:111-138, 1964.

[27] T. T. Wu, C. N. Yang. Concept of nonintegrable phase factors and global formulation of gauge fields. Phys. Rev. D 12:3845-3857, 1978. DOI:10.1103/PhysRevD.12.3845 